\title{
A Laplace Equation Approach for Shape Comparison
}

\author{
Eric Pichon ${ }^{a}$ and Delphine Nain $^{b}$ and Marc Niethammer ${ }^{a}$ \\ ${ }^{a}$ Department of Electrical Engineering, Georgia Institute of Technology, Atlanta GA 30332, \\ USA \\ ${ }^{b}$ College of Computing, Georgia Institute of Technology, Atlanta GA 30332, USA
}

\begin{abstract}
In this paper we propose a principled approach for shape comparison. Given two surfaces, one to one correspondences are determined using the Laplace equation. The distance between corresponding points is then used to define both global and local dissimilarity statistics between the surfaces. This technique provides a powerful method to compare shapes both locally and globally for the purpose of segmentation, registration or shape analysis. For improved accuracy, we propose a Boundary Element Method. Our approach is applicable to datasets of any dimension and offers subpixel resolution. We illustrate the usefulness of the technique for validation of segmentation, by defining global dissimilarity statistics and visualizing errors locally on color-coded surfaces. We also show how our technique can be applied to multiple shapes comparison.
\end{abstract}

\section{INTRODUCTION}

The ability to quantify both local and global differences between shapes is an important step in computer aided medical diagnosis.

After imaging and segmentation have been performed, shape comparison techniques can be used to determine the deviation some structure or organ has to some standard template. Such differences in shape can be used for pathology diagnostic. For example, abnormal brain ventricle shape is a symptom of schyzophrenia.

Another application of shape comparison is the validation of image segmentation techniques. The diversity of proposed segmentation algorithms raises the issue of performance evaluation. For a given organ, which algorithm results in the most accurate segmentation? How can its robustness be quantified? Using a measure of shape similarity, the performance of a particular algorithm can be quantitatively assessed by comparing its output to a known ground truth, a manual segmentation or the output of another segmentation algorithm.

In this paper we describe a mathematical methodology to address the problem of shape comparison by determining one-to-one correspondences between two shapes and defining both global and local similarity statistics. To compare more than two shapes, statistics are computed with reference to a mean shape.

Section 2 motivates our approach. In Section 3 we introduce the Laplace equation and propose different methods to solve it. In Section 4 we show how this can be used to define corresponding points between two shapes. We also show how to compare more than two shapes by using a mean shape. In Section 5 we illustrate our method for the purpose of validation of image segmentation. We show how to visualize locally the dissimilarity between the surfaces allowing the user to analyze the accuracy of a segmentation in an anatomically meaningful way. We also demonstrate how global metrics can be derived to quantify the accuracy of the segmentation. The complete methodology is illustrated on synthetic and real datasets.

Further author information: (Send correspondence to:)

Eric Pichon: E-mail: eric@ece.gatech.edu, Delphine Nain : E-mail: delfin@cc.gatech.edu, Marc Niethammer: E-mail: marcn@ece.gatech.edu

Medical Imaging 2006: Visualization, Image-Guided Procedures, and Display, edited by Kevin R. Cleary, Robert L. Galloway, Jr., Proc. of SPIE Vol. 6141, 614119, (2006) 1605-7422/06/\$15 - doi: $10.1117 / 12.651135$ 


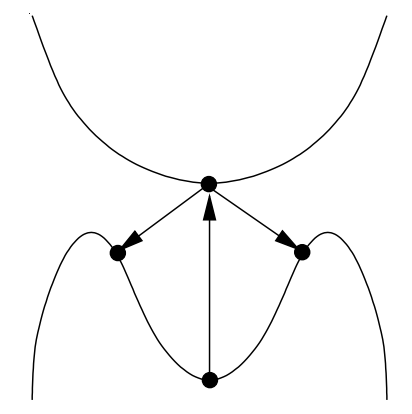

Figure 1. The minimal Euclidean distance between points may lead to correspondences that are not unique and not symmetric.

\section{MOTIVATION AND RELATED WORK}

This paper is concerned with shape comparison, i.e., with quantifying similarities between shapes. Similarities may be defined on different levels of resolution, ranging from global shape metrics to local correspondences. A classical example for a global shape metric is the symmetric Hausdorff distance, measuring the maximal minimum Euclidean distance between two sets. While it provides global insight with regards to the "worst-case-deviation" between two shapes, the symmetric Hausdorff distance (as an example of global shape metric) fails to resolve local shape variations. See ${ }^{1}$ for an overview of shape matching techniques.

This paper aims at calculating shape differences on a local level for descriptional flexibility. These local measures of shape differences may then be interpreted directly or may be used to compute more global shape similarity metrics.

We represent a shape by a closed hyper-surface: e.g., a closed curve in the plane or a closed surface in three-dimensional space. Defining a local shape metric then hinges (in the most local case) on establishing point to point correspondences between surfaces. A method to obtain surface correspondence points should be computationally efficient, should generalize to different dimensions, and should yield a correspondence map that is symmetric, one-to-one and ideally also continuous (i.e., homeomorphic). Figure (1) shows some exemplary correspondences between two curves based on the smallest Euclidean distance from curve to curve and highlights the desirability of correspondences that are symmetric and one-to-one.

We advocate a Laplace equation based approach to find point correspondences between two surfaces with the aforementioned properties. This is a natural approach for the following reason: given two surfaces $\Sigma_{1}$ and $\Sigma_{2}$, envision a flow field transporting particles starting on surface $\Sigma_{1}$ to $\Sigma_{2}$. The conditions of (i) symmetry, (ii) one-to-one correspondence and (iii) continuity are in this setting equivalent to requiring that (i) the flow can be reversed, (ii) a particle does not get caught up in a vortex or at a stagnant point (unless it started there), and (iii) particles that start close to each other on $\Sigma_{1}$ arrive "close to each other" on $\Sigma_{2}$. Condition (ii) yields a potential flow, i.e., the flow field $\boldsymbol{v}$ can be expressed as the gradient of a scalar potential $u, \boldsymbol{v}=\nabla u$. If the fluid is assumed to be incompressible, $\boldsymbol{v}$ needs to be divergence free as well, i.e., $\nabla \cdot \boldsymbol{v}=0$, but this implies $\nabla \cdot \nabla u=\Delta u=0$, which is Laplace's equation. Solving Laplace's equation (with suitable boundary conditions) between $\Sigma_{1}$ and $\Sigma_{2}$ will induce point-to-point correspondences between $\Sigma_{1}$ and $\Sigma_{2}$ through the streamlines of $\boldsymbol{v}$. Figure (2) shows the vector fields induced by a distance function of a cavity and by the solution of Laplace's equation. All particles starting on the boundary of the cavity and moving along the vector field induced by the solution of Laplace's equation will eventually leave the cavity. This is not true for all particles for the vector field induced by the distance function.

Laplace equation approaches have previously been used $\mathrm{in}^{2,3}$ for colon surface flattening and centerline extraction and in ${ }^{4,5}$ for thickness measurements. This paper introduces a framework to measure local shape variability. Given multiple surfaces $\Sigma$ and a mean (or comparison) surface $\Sigma_{m}$ point correspondences are used to measure local distance differences. The local distances between surfaces can then be used for visualization 


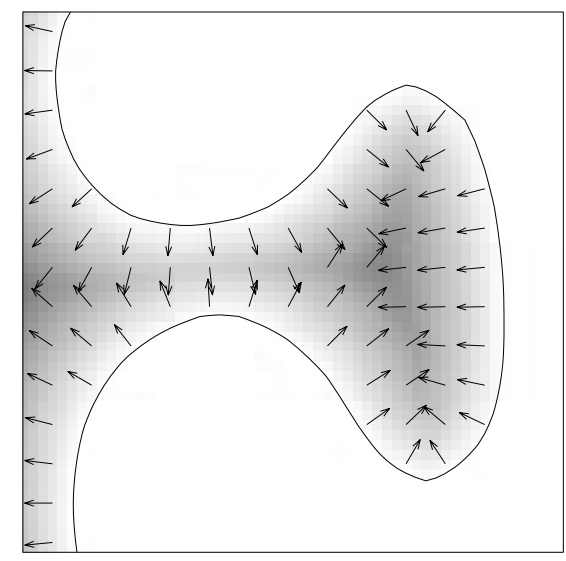

(a) Vector field induced by the distance function.

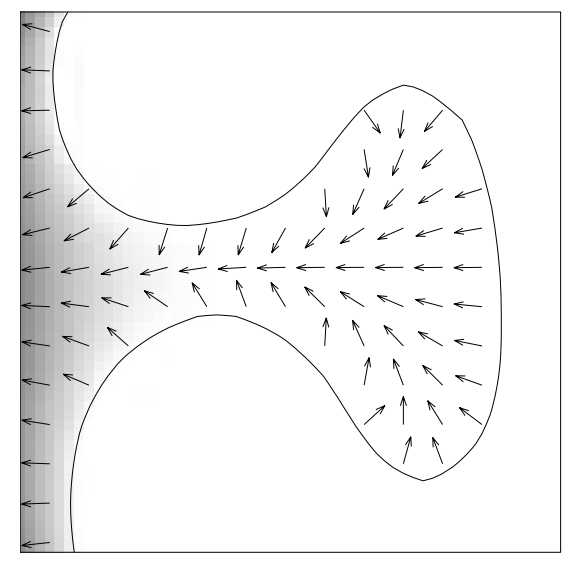

(b) Vector field induced by the solution of Laplace's equation.

Figure 2. Vector fields induced by the distance function and by the solution of Laplace's equation. Dark values indicate larger solution values.

purposes (e.g., to color-code the mean shape) and to define local and global statistics. Statistics may be computed at a single point of a mean surface over all surfaces $\Sigma$ to assess local shape variation. Alternatively one can compute statistical measures of distance variations over a single surface. The latter is particularly useful in the context of validation of surface segmentations: how good is a segmentation compared to a given ground truth.

\section{LAPLACE EQUATION}

Assume that we have two regions $R_{1}$ and $R_{2}$ whose boundaries $\Sigma_{1}$ and $\Sigma_{2}$ are surfaces implicitly represented as zero level sets of functions $\phi_{1}$ and $\phi_{2}$, i.e.,

$$
\Sigma_{i}=\partial R_{i}=\left\{\mathbf{x} / \phi_{i}(\mathbf{x})=0\right\}, i \in\{1,2\} .
$$

Such a representation is natural for partial differential equations based segmentation algorithms. Regions defined by binary maps and triangulated surfaces can also be represented using level sets.

The Laplace equation

$$
\begin{cases}\nabla^{2} u(\mathbf{x})=0 & \mathbf{x} \in R_{1} \ominus R_{2} \\ u(\mathbf{x})=1 & \mathbf{x} \in \Sigma_{1} \\ u(\mathbf{x})=-1 & \mathbf{x} \in \Sigma_{2}\end{cases}
$$

underlies Fourier's law of heat conduction. In this context $u$ can be interpreted as a temperature. Equation (2) describes the steady state temperature profile $u$ in between the surfaces (here, $R_{1} \ominus R_{2} \triangleq\left(R_{1} \backslash R_{2}\right) \cup\left(R_{2} \backslash R_{1}\right)$ is the symmetric difference of the two regions) resulting from prescribed boundary conditions on $\Sigma_{1}$ and $\Sigma_{2}$. The field $u$ is harmonic. It is smooth and its derivatives of all order exist. ${ }^{6}$ Moreover the gradient does not vanish and therefore the vector field

$$
\mathbf{v}=\frac{\nabla u}{\|\nabla u\|}
$$

is also smooth. By following $\mathbf{v}$ (resp. $-\mathbf{v})$ starting at a point on $\Sigma_{1}\left(\right.$ resp. $\Sigma_{2}$ ) we are assured to reach a unique point on $\Sigma_{2}$ (resp. $\Sigma_{1}$ ). This is a very desirable property for establishing one-to-one point correspondences between $\Sigma_{1}$ and $\Sigma_{2}$. A variety of schemes exist to solve (2) based on gradient descent (the Heat equation) or superposition of fundamental analytic solutions (Boundary Element Method). 


\subsection{Heat Equation}

Instead of solving the steady state problem (2) directly, we can use the heat equation

$$
\frac{\partial u}{\partial t}=\nabla^{2} u
$$

which with appropriate boundary conditions will converge to the solution of (2). In the simplest possible case, we discretize the time derivative using an Euler forward or backward approximation and the spatial derivatives using central differences.

\subsection{Boundary Element Method (BEM)}

The level set representation yields subpixel accuracy. Using subpixel boundary information is not straightforward with finite difference based schemes. In BEM, the boundary is approximated by $N$ elements and the solution is constructed based on the superposition of the fundamental solutions of the Laplace equation for each boundary element. $^{7}$ For simple boundary parameterizations, analytical solutions exist. Furthermore, the boundary element method allows for analytical gradient computations which is very useful when calculating (3). ${ }^{8,9}$

The BEM is a powerful and accurate method for solving the Laplace equation on the usually complicated domains resulting from the segmentation of medical datasets. A 2D synthetic example is shown on Fig. 3 along with a zoom-in on the solution of the Laplace equation with the two proposed methods. The BEM is not restricted to the underlying grid and can take full advantage of the subpixel accuracy of the boundaries. It therefore outperforms the simple finite difference approach. More details can be found in Appendix 6 .

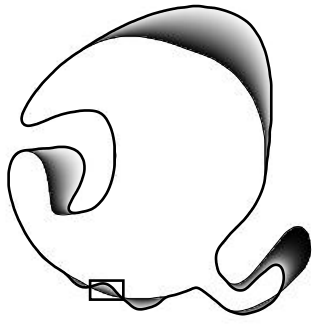

(a) Solution of the Laplace equation on $R_{2} \ominus R_{1}$.

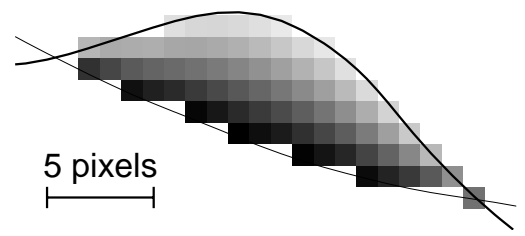

(c) Finite difference method (Heat equation).

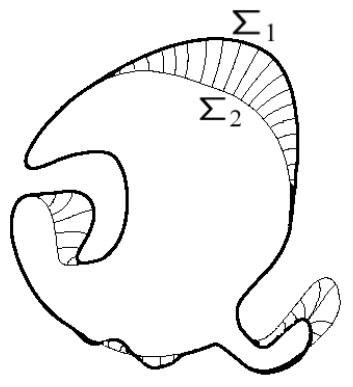

(b) Streamlines defined from the gradient of the solution of the Laplace equation.

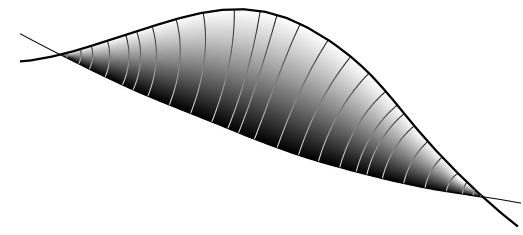

(d) Boundary element solution with streamlines of the gradient field.

Figure 3. 2D synthetic example and zoom in on rectangular region. 


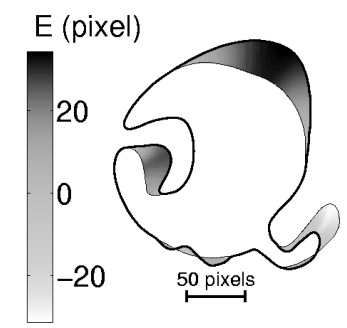

Figure 4. Local dissimilarity for a 2D synthetic example.

\section{DEFINING A DISTANCE BETWEEN SHAPES}

\subsection{Local Dissimilarity}

As explained in the previous section from any point $s$ on surface $\Sigma_{1}$, (resp. $\left.\Sigma_{2}\right)$, a trajectory can be determined to $\Sigma_{2}$, (resp. $\Sigma_{1}$ ), by following the characteristics (i.e., the gradient) of the Laplace equation. A particle can be moved from one surface to the other according to

$$
\begin{cases}s_{t}(t)=-\nabla u \circ s(t) & s \in \Sigma_{1} \\ s_{t}(t)=+\nabla u \circ s(t) & s \in \Sigma_{2} \\ s(0)=s & \end{cases}
$$

As explained previously such correspondence are well defined, one to one and symmetric.

To each point $s$ on one of the two surfaces we can therefore associate the length $l$ to the corresponding point $s^{\prime}$ on the other surface. We propose to define the local error $E$ as $-l$ where $\Sigma_{1}$ is inside $\Sigma_{2}$ and $+l$ otherwise. This scalar field is defined on both surfaces and describes locally the dissimilarity between the surfaces. See Figure 4.1 for an illustration on a synthetic example.

\subsection{Visualization}

The surfaces $\Sigma_{1}$ and $\Sigma_{2}$ (or any surface lying in between those) can be colored with the local dissimilarity scalar field $E$ (Fig. 6(a), 6(b), 6(c)). We use a perceptually linear color scale as proposed by Lefkowitz et al.. ${ }^{10}$

\subsection{Metrics}

We define a probability space $\left(\Sigma_{1} \cup \Sigma_{2}, P\right)$ on the union of the surfaces by $P(S)=\frac{\mathcal{A}(S)}{\mathcal{A}\left(\Sigma_{1}\right)+\mathcal{A}\left(\Sigma_{2}\right)}$ where $\mathcal{A}(S)$ is the area of an element of surface $S \subset \Sigma_{1} \cup \Sigma_{2}$. The local dissimilarity field $E$ can then be interpreted as a random variable.

The statistics of $E$ can be used to define several metrics that will quantify the global dissimilarity between the surfaces.

- $\sup (|E|)$ is the largest distance between corresponding points of $\Sigma_{1}$ and $\Sigma_{2}$. This information is equivalent to the Hausdorff distance. Moreover, $\inf (E)$ and $\sup (E)$ are the largest under and over-segmentation errors. The quantiles of $E$ convey the same information as the partial Hausdorff distance.

- mean $(|E|)$ is the average distance between the surfaces.

- $P(E \leq-3)$ is the probability that $\Sigma_{1}$ would be inside $\Sigma_{2}$ by 3 units.

- the probability density function $p_{E}$ of $E$ gives the full error distribution.

See Table 1 for the values of these statistics on the synthetic and brain datasets. Additional metrics can easily be defined. For example, the expectancy of $1 /\left(1+p E^{2}\right)$ would be equivalent to Pratt's Figure of Merit. ${ }^{11}$ 


\begin{tabular}{c|cc|c|c|c|c} 
& $\sup (E)$ & $\begin{array}{c}99 \% \\
\text { percentile }\end{array}$ & $\inf (E)$ & $\begin{array}{c}1 \% \\
\text { percentile }\end{array}$ & mean $(|E|)$ & $P(E \leq-3)$ \\
\hline Synthetic (pixel) & 31.42 & 31.24 & -28.21 & -20.40 & 5.32 & $9.50 \%$
\end{tabular}

Table 1. Global statistics of the local dissimilarity $E$ on the synthetic example.

\subsection{Comparing $n$ surfaces}

In order to study the local variation in a set of $n$ surfaces defined by the level sets function $\phi_{1} \ldots \phi_{n}$, the level set function of the mean shape is defined to be

$$
\phi_{m} \triangleq \sum_{i=1}^{n} \phi_{i}
$$

and all $n$ shapes are compared to the mean surface.

Figure 4.4 shows the local dissimilarity to a mean shape for $n=8$ prostates. Surfaces were segmented from pre-operative 1.5T MR images acquired using an endorectal coil integrated with a pelvic multi-coil (Signa LX, GE Medical systems, Milwaukee, WI). The slice thickness is of $3 \mathrm{~mm}$ with a slice gap of $0 \mathrm{~mm}$, matrix of $256 \times 192$.

\section{VALIDATION FRAMEWORK}

The previously defined formalism can be applied directly to the quantitative validation of segmentation if the ground truth is known. In that case, one of the surfaces is set to be the segmentation result and the other the ground truth. The dissimilarity between the segmentation and the ground truth can then be compared both quantitatively and qualitatively. If the ground truth is not available, the results of segmentations obtained through different techniques can be compared (e.g. automatic and human expert).

In ${ }^{12}$ Zhang reviews classical validation metrics. These discrepancy metrics are based either on the sole frequency of mis-segmented voxels (e.g, the Dice similarity coefficient) or on their position via some sort of distance information (e.g., the Hausdorff distance, Yasnoff's Normalized Discrepancy etc.). Typically no distinction is made between under and over-segmentation and the Euclidean distance is employed and, as in the case of the Hausdorff distance, ill-defined underlying correspondences between the ground truth and the segmentation are implicitly used. Moreover many of these metrics are dimensionless scalars whose interpretation can be problematic.

In contrast the error distance $E$ discriminates between under and over-segmentation (negative values of the local dissimilarity scalar field $E$ will correspond to under-segmentation and positive values will correspond to over-segmentation). Moreover it is based on well-defined, physically grounded correspondences and has the dimension of a distance.

Here, as an illustration we use, as the ground truth, the expert manual segmentations of a full brain and a tumor from the Brain Tumor Database ${ }^{13}$ and the output of a previously proposed algorithm ${ }^{14}$ as the segmentation, see Figures 6 and 7 and Table 2.

\begin{tabular}{c|cc|cc|c|c} 
& $\sup (E)$ & $\begin{array}{c}99 \% \\
\text { percentile }\end{array}$ & $\inf (E)$ & $\begin{array}{c}1 \% \\
\text { percentile }\end{array}$ & mean $(|E|)$ & $P(E \leq-3)$ \\
\hline Brain $(\mathrm{mm})$ & 14.08 & 7.09 & -86.10 & -11.87 & 2.31 & $4.03 \%$ \\
\hline Tumor $(\mathrm{mm})$ & 6.83 & 5.15 & -4.75 & -3.18 & 1.58 & $1.17 \%$
\end{tabular}

Table 2. Global statistics of the local dissimilarity $E$ on the brain and tumor examples. 


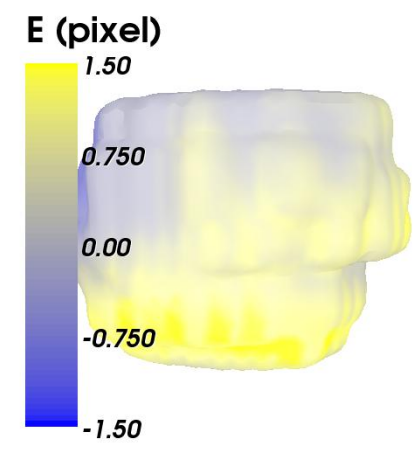

(a)

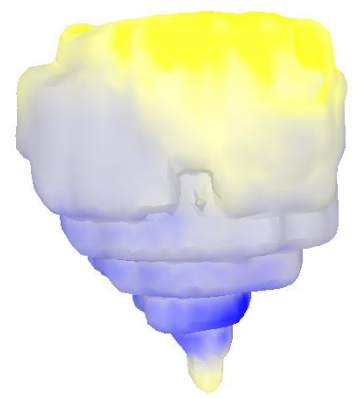

(d)

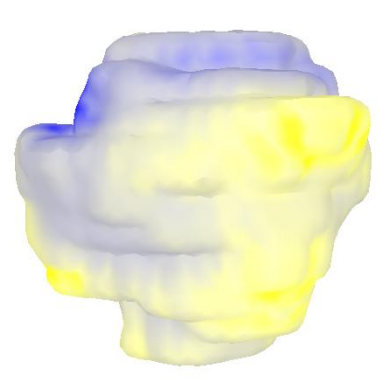

(g)

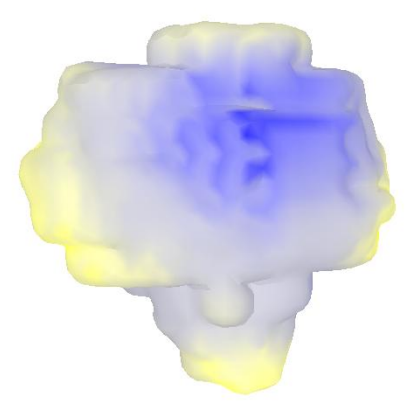

(b)

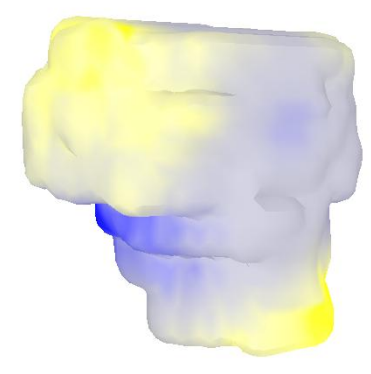

(e)

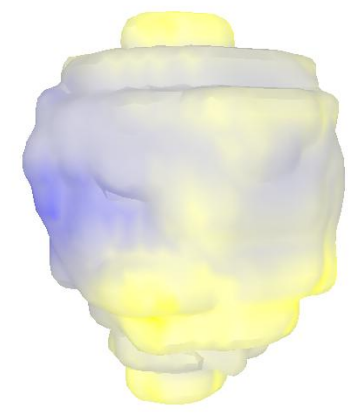

(h)

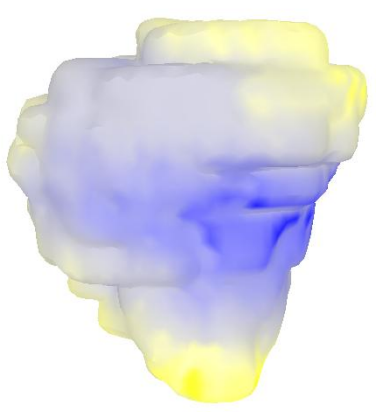

(c)

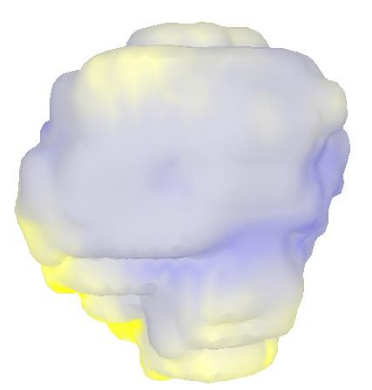

(f)

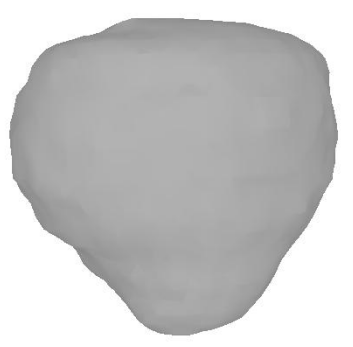

(i) Mean shape

Figure 5. Comparison of $n=8$ prostates to a mean surface. These are color images.

In the proposed brain segmentation a large portion of the left hemisphere was left out (under-segmented) and the superior sagittal and transverse sinuses (venous channels in the back of the brain) were erroneously classified as brain (over-segmented). This visualization scheme allows researchers and clinicians to interpret the performance of a segmentation in an anatomically meaningful way. 


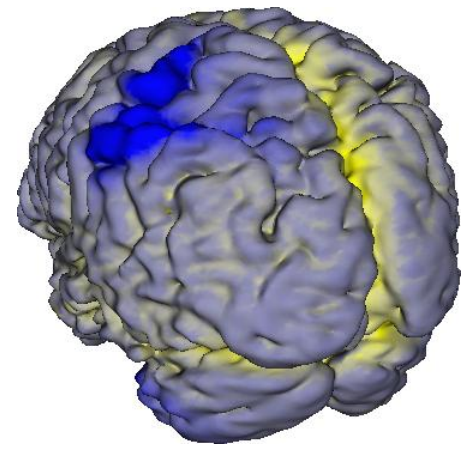

(a) Ground Truth

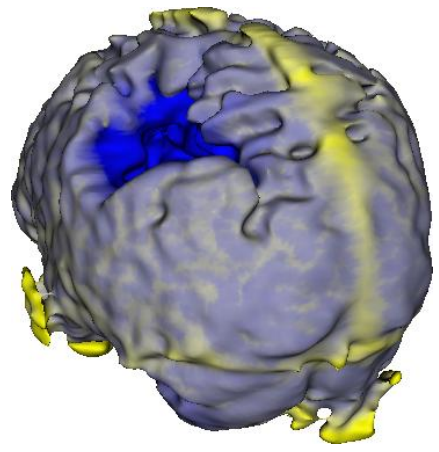

(b) Segmentation
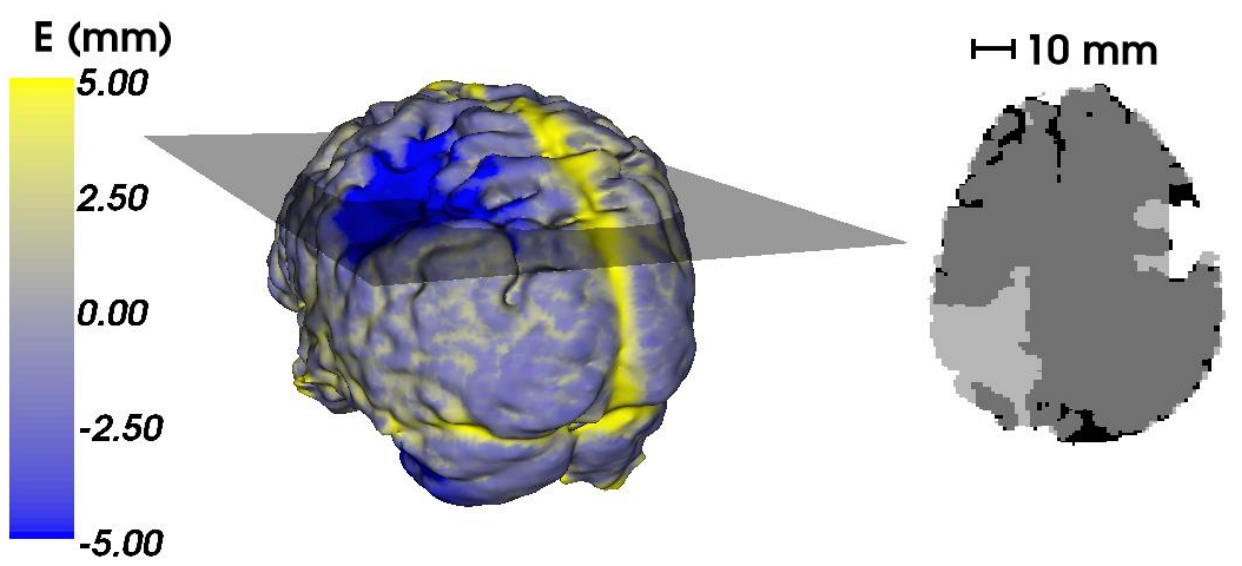

(c) The underlying ground truth and segmentation on the gray slice are shown on the right. Over-segmented regions are in dark gray, under-segmented regions are in light gray.

Figure 6. Real dataset of a full brain The surfaces are colored with the error distance $E$ using a perceptually linear color scale. ${ }^{10}$ These are color images.

\section{CONCLUSION}

We proposed a novel technique for surface comparison as well as a probabilistic framework that extends distancebased shape comparison metrics. Our approach is based on constructing one-to-one correspondences between the two shapes under study. The correspondences are based on the Laplace equation. which can be solved numerically by iterating the Heat equation or analytically using a Boundary Element Method. The method can be applied to datasets of any dimension and offers subpixel resolution. We believe that the general concepts outlined in this paper could also prove useful for other applications such as shape analysis and non-rigid deformations.

\section{Acknowledgements}

This work was supported in part by NIH (NAC and NAMIC). The authors would like to thank Clare Tempany MD, Agnieska Szot MD, Jianqing Zhang MD and Steven Haker PhD of the Brigham and Women's Hospital for the prostate datasets as well as Gabriel Wardi of Georgia Tech for his help with the implementation. 


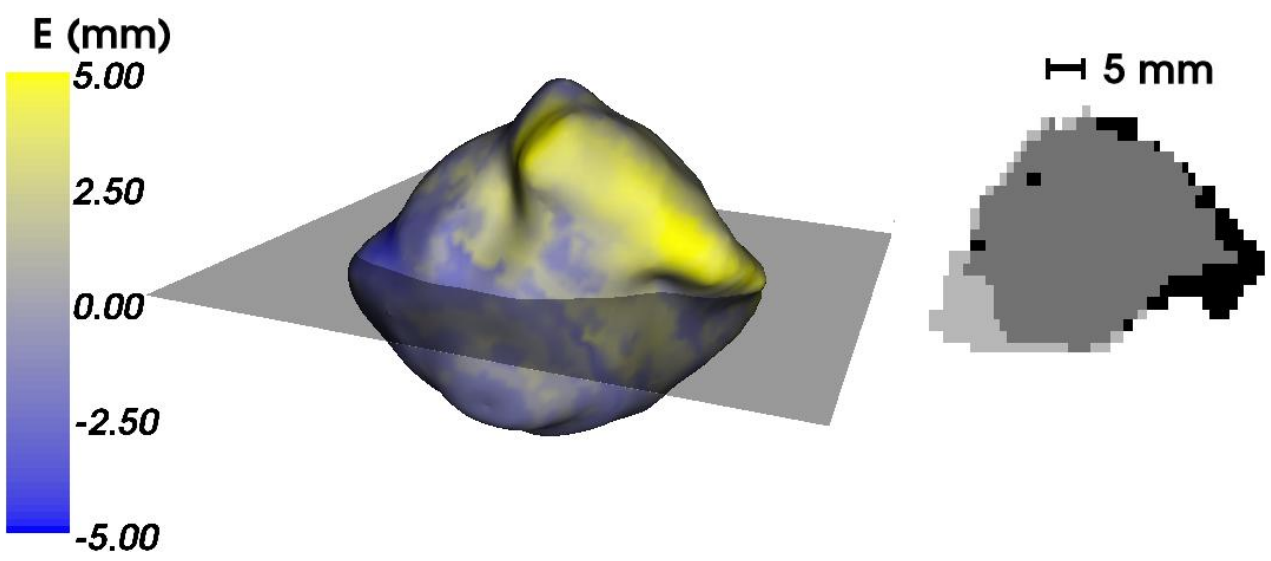

Figure 7. Real dataset of a tumor. The surfaces are colored with the error distance $E$ using a perceptually linear color scale. ${ }^{10}$ These are color images.

\section{Appendix}

For an approximate boundary element solution of (2) we start with the weighted residual formulation

$$
\int_{R}\left(\nabla^{2} \tilde{u}\right) w_{l} \mathrm{~d} R \equiv \int_{R} \epsilon_{R} w_{l} \mathrm{~d} R=0
$$

where $\tilde{u}$ is the approximate solution, $w_{l}$ is the weighting function, $\epsilon_{R}$ denotes the error, and $R=R_{\mathrm{SEG}} \ominus R_{\mathrm{GT}}$ is the symmetric difference* of the sets $R_{\mathrm{SEG}}$ and $R_{\mathrm{GT}}$. Applying Green's theorem to (7) twice yields ${ }^{7}$

$$
\int_{R}\left(\nabla^{2} w_{l}\right) \tilde{u} \mathrm{~d} R=\int_{\Sigma} \frac{\partial w_{l}}{\partial \boldsymbol{n}} \tilde{u}-\frac{\partial \tilde{u}}{\partial \boldsymbol{n}} w_{l} \mathrm{~d} \Sigma .
$$

If we choose $w_{l}$ as the fundamental solution of $(2)$ centered at $\boldsymbol{x}_{l}$, i.e., $\nabla^{2} w_{l}=\delta(r)$, where $r:=\left\|\boldsymbol{x}-\boldsymbol{x}_{l}\right\|$, and take into account that $\boldsymbol{x}_{l}$ might be a boundary point, we obtain

$$
c_{l} \tilde{u}\left(\boldsymbol{x}_{l}\right)=\int_{\Sigma} \frac{\partial \tilde{u}}{\partial \boldsymbol{n}} w_{l}-\tilde{u} \frac{\partial w_{l}}{\partial \boldsymbol{x}_{l}} \mathrm{~d} \Sigma
$$

where the fundamental solution $w_{l}$ is given as

$$
w_{l}=-\frac{1}{2 \pi} \ln r \quad \text { in dimension } 2, \quad w_{l}=\frac{1}{4 \pi r} \quad \text { in dimension } 3,
$$

and (for planar boundary elements)

$$
c_{l}=0 \quad \text { if } \boldsymbol{x}_{l} \in(R \cup \Sigma)^{c}, \quad c_{l}=\frac{1}{2} \quad \text { if } \boldsymbol{x}_{l} \in \Sigma, \quad c_{l}=1 \quad \text { otherwise. }
$$

The boundary $\Sigma$ is approximated by $N$ boundary elements $\Sigma_{i}, i=1,2, \ldots, N$ (i.e., either line segments or triangular patches) with $\tilde{u}$ and $\frac{\partial \tilde{u}}{\partial \boldsymbol{n}}$ constant on each such element ${ }^{\dagger}$. We can then write

$$
\sum_{i=1}^{N} \tilde{q}_{i} \int_{\Sigma_{i}} w_{l} \mathrm{~d} \Sigma_{i}=c_{l} \tilde{u}\left(\boldsymbol{x}_{l}\right)+\sum_{i=1}^{N} \tilde{u}_{i} \int_{\Sigma_{i}} \frac{\partial w_{l}}{\partial \boldsymbol{n}} \mathrm{d} \Sigma_{i}
$$

The integrals in (8) can be computed analytically. ${ }^{8,9}$ We define

$$
g_{l i}=\int_{\Sigma_{i}} w_{l} \mathrm{~d} \Sigma_{i}, \quad h_{l i}=\int_{\Sigma_{i}} \frac{\partial w_{l}}{\partial \boldsymbol{n}} \mathrm{d} \Sigma_{i}, \quad G=\left(g_{l i}\right), \quad H=\left(h_{l i}\right) .
$$

${ }^{*} A \ominus B=(A \backslash B) \cup(B \backslash A)$

${ }^{\dagger}$ More accurate parametrizations exist. 
In our case, only temperature values, $\tilde{u}$, are prescribed on the boundary $\Sigma$. It remains to determine the $N$ unknowns, $\tilde{q}_{i}$, by (8). If we pick the $N$ points $\boldsymbol{x}_{l}$ as the centers of gravity of the $N$ boundary elements, compute $G$ and $H$ based on these points and stack the values for $\tilde{q}_{i}$ and $\tilde{u}_{i}$ in the two vectors $\boldsymbol{q}$ and $\boldsymbol{u}$ respectively, we obtain the matrix equation

$$
G \boldsymbol{q}=\left(\frac{1}{2} I+H\right) \boldsymbol{u}
$$

Solving (9) for $\boldsymbol{q}$ yields the complete boundary information. Then, every value $\tilde{u}$ in the interior of $R$ can easily be obtained by

$$
\tilde{u}(\boldsymbol{x})=\sum_{i=1}^{N} \tilde{q}_{i} g_{l i}-h_{l i} \tilde{u}_{i}
$$

The gradient is then given by

$$
\nabla \tilde{u}(\boldsymbol{x})=\sum_{i=1}^{N} \tilde{q}_{i} \int_{\Sigma_{i}} \nabla_{\boldsymbol{x}_{l}} w_{l} d \Sigma_{i}-\tilde{u}_{i} \int_{\Sigma_{i}} \nabla_{\boldsymbol{x}_{l}}\left(\frac{\partial w_{l}}{\partial \boldsymbol{n}}\right) d \Sigma_{i}
$$

Also, all integrals in (10) are solvable analytically. 8,9

\section{REFERENCES}

1. R. C. Veltkamp, "Shape matching: similarity measures and algorithms," Tech. Rep. UU-CS-2001-03, Utrecht University, 2001.

2. S. Haker, S. Angenent, A. Tannenbaum, and R. Kikinis, "Nondistorting flattening maps and the 3D visualization of colon CT images," IEEE Trans. on Medical Imaging 19, pp. 665-670., 2000.

3. D. Nain, S. Haker, W. Eric L. Grimson, E. Cosman Jr, W.W. Wells, H. Ji, R. Kikinis and C.F. Westin, "Intra-patient prone to supine colon registration for synchronized virtual colonoscopy," in MICCAI, 2002.

4. S. Jones, B. Buchbinder, and I. Aharon, "Three-dimensional mapping of cortical thickness using Laplace's equation," Human Brain Mapping 11, pp. 12-32, 2000.

5. A. Yezzi and J. L. Prince, "A PDE approach for measuring tissue thickness," in CVPR, 1, pp. 87-92, 2001.

6. L. Evans, Partial Differential Equations, vol. 19 of Graduate Studies in Mathematics, American Mathematical Society, 1998.

7. G. Gipson, Boundary Element Fundamentals - Basic Concepts and Recent Developments in the Poisson Equation, Computational Mechanics Publications, 1987.

8. T. Kuwabara and T. Takeda, "Boundary element method using analytical integration for three-dimensional Laplace problem," Transactions of the Institute of Electrical Engineers of Japan 106(6), pp. 25-31, 1986.

9. M. Fratantonio and J. J. Rencis, "Exact boundary element integrations for two-dimensional Laplace equation," Engineering Analysis with Boundary Elements 24, pp. 325-342, 2000.

10. H. Lefkowitz and G. Herman, "Color scales for image data," IEEE Computer Graphics and Applications 12(1), pp. 72-80, 1992.

11. W. Pratt, Digital Image Processing, Wiley-Interscience, 1978.

12. Y. Zhang, "A survey on evaluation methods for image segmentation," Pattern Recognition 29(8), pp. 1335-1346, 1996.

13. M. Kaus, S. Warfield, A. Nabavi, P. Black, F. Jolesz, and R. Kikinis, "Automated segmentation of MRI of brain tumors," Radiology 218(2), pp. 586-591, 2001.

14. E. Pichon, A. Tannenbaum, and R. Kikinis, "A statistically based surface evolution method for medical image segmentation: presentation and validation," in MICCAI, 2, pp. 711-720, 2003. 